\title{
Molecular characterisation of Leptospira strains in Pakistan
}

\author{
Muhammad Luqman Sohail ${ }^{1}$, Muhammad Sarwar Khan ${ }^{1}$, Muhammad Avais ${ }^{1}$, \\ Muhammad Yasir Zahoor ${ }^{2}$, Irfan Khattak $^{3}$, Aqeela Ashraf ${ }^{2}$, Omer Naseer ${ }^{1}$ \\ ${ }^{1}$ Department of Clinical Medicine and Surgery, \\ ${ }^{2}$ Institute of Biochemistry and Biotechnology, \\ ${ }^{3}$ Department of Epidemiology and Public Health, \\ University of Veterinary and Animal Sciences, 54000 Lahore, Pakistan \\ vet.luqman@gmail.com
}

Received: May 25, $2016 \quad$ Accepted: November 7, 2016

\begin{abstract}
Introduction: Leptospirosis affects a wide range of mammals, humans, and even a few poikilothermic animal species. In Pakistan, serological studies of equine leptospirosis have reported a prevalence of over $40 \%$, but no study has ever been conducted towards molecular detection of Leptospira in horses. Material and Methods: Blood samples from 128 horses were screened using ELISA and 41 positive samples were examined for the presence of leptospiral DNA using specific primers for 16S rRNA gene. Results: Out of 41 tested samples, 20 samples were found to be PCR-positive, revealing a fragment of $306 \mathrm{bp}$ after gel electrophoresis. Sequencing and phylogenetic analysis of positive samples revealed circulation of pathogenic Leptospira spp. in Pakistani horses. No evidence of circulation of intermediate species was found in this study. Conclusion: This study reports the first molecular evidence of equine leptospirosis in Pakistan and lays ground for further research in this area. It also confirms the efficiency of $16 \mathrm{~S}$ rRNA for the diagnosis of equine leptospirosis.
\end{abstract}

Keywords: horses, Leptospira interrogans, 16S rRNA, Pakistan.

\section{Introduction}

Leptospirosis is an infectious disease caused by spirochaete in the genus Leptospira along with its more than 260 serovars (24), infecting a variety of domestic and wild animals (1). It has also been reported in a few poikilothermic vertebrates such as amphibians and reptiles (11). Leptospirosis is one of the six diseases enlisted by OIE with reference to "animal diseases and climate change" (19). The disease is most commonly found in rural and urban areas of tropical and subtropical parts of the world (30). It spreads more readily in tropical regions as Leptospira can survive longer in warm and humid environment. Hence, there are areas in Southeast Asia, China, South and Central America, and Africa where leptospirosis is endemic (25).

Leptospirosis usually evolves as an acute or chronic disease, affecting individual animals or animal groups. In horses, leptospirosis is displayed through equine recurrent uveitis and reproductive disorders including the third trimester abortion. Most infections are asymptomatic (9). Early and accurate diagnosis is essential to cure leptospirosis (10). The serological methods are most commonly used to diagnose leptospirosis (13). The microscopic agglutination test (MAT) and ELISA have pivotal role in veterinary diagnosis $(16,26)$. However, serological testing is complicated because of the high degree of crossreactivity between various Leptospira serovars (17). Recently, molecular techniques such as conventional and real-time PCR are recognised as specific and sensitive tests for the rapid detection of infection during early stages of the disease and often negate the need for isolation and culture of the infecting organism for a confirmatory result. These techniques can be performed reliably on various templates, including blood, urine, and kidney tissues (12). Rapid and accurate results using molecular diagnostic techniques are now replacing serological tests in areas where leptospirosis is endemic (17). 
In Pakistan, serological studies on equine leptospirosis showed a prevalence of up to $44 \%$ in some areas of the country, which is quite alarming (21). All previous studies reporting Leptospira in Pakistan were based on serological testing $(2,20)$. However, no attempts had ever been made at molecular detection of the organism in any mammalian host. Therefore, This fact encouraged us to diagnose equine leptospirosis using molecular techniques for the first time in Pakistan. Consequently, the presence of leptospiral DNA in horses was confirmed.

\section{Material and Methods}

Sample collection. A total of 128 blood samples were collected from horses of rural and urban areas of Punjab, Pakistan. The blood was aseptically collected by jugular vein puncture into EDTA-coated vacutainers (BD Biosciences, USA).

All collected samples were subjected to ELISA (Horse Leptospira IgG ELISA Kit, MyBiosource, USA) to screen for equine leptospirosis. Out of 128 samples, 41 samples were ELISA positive and consequently examined for the presence of leptospiral DNA by PCR.

Genomic DNA extraction. Genomic DNA was extracted from the blood samples using PureLink ${ }^{\circledR}$ Genomic DNA minikit (Thermo Fisher Scientific Inc. USA) according to the manufacturer's instructions. Concentration of extracted DNA was measured using Nanodrop-1000 spectrophotometer (Thermal Scientific, USA).

PCR amplification of Leptospira 16S rRNA gene. The amplification of Leptospira 16s rRNA gene was performed using primers Lp-F: 5'GCGCGTCTTAAACATGCAAG -3' and Lp-R: 5'CTTAACTGCTGCCTCCCGTAG-3' (7). PCR amplification was performed with $50 \mu \mathrm{L}$ volume containing: $1 \mu \mathrm{g}$ of template DNA, $1 \mu \mathrm{M}$ of each forward and reverse primers, $2 \mathrm{mM} \mathrm{MgCl}_{2}, 200$ $\mu \mathrm{MdNTP}, 5 \mu \mathrm{L}$ of $10 \times$ PCR buffer, and 1 unit of Taq DNA polymerase (Invitrogen, USA). A master cycler gradient was used with initial denaturation at $95^{\circ} \mathrm{C}$ for $5 \mathrm{~min}$, followed by 30 cycles of denaturation at $94^{\circ} \mathrm{C}$ for $1 \mathrm{~min}$, annealing for $1 \mathrm{~min}$ at $58^{\circ} \mathrm{C}$, and extension at $72^{\circ} \mathrm{C}$ for $1 \mathrm{~min}$, followed by a final extension for $5 \mathrm{~min}$ at $72^{\circ} \mathrm{C}$.

Agarose gel electrophoresis. PCR product was analysed on $1 \%(\mathrm{wt} / \mathrm{vol})$ agarose gel. DNA ladder of $1 \mathrm{~Kb}$ was used as a molecular marker. The expected size of amplicons was $306 \mathrm{bp}$.

Sequencing and phylogenetic analysis of $16 \mathrm{~S}$ rRNA gene. Amplified fragments were gel-purified using an EZ-10 Spin Column DNA Gel Extraction Kit (Bio Basic Inc, USA) according to manufacturer's instructions. Sequencing of DNA fragments was performed using dideoxy chain-termination method. The 16S rRNA gene sequences of the isolates of
Leptospira were retrieved from NCBI database. Phylogenetic tree was constructed based on partial nucleotide sequence of $16 \mathrm{~S}$ rRNA gene using Molecular Evolutionary Genetics Analysis (MEGA) version 6 (www.megasoftware.net). The tree topologies were evaluated using bootstrap test of phylogeny with maximum likelihood obtained after 1000 replicates of the dataset.

\section{Results}

Out of 41 tested samples, $20(30.76 \%)$ samples were found to have DNA of Leptospira. Primers designed for leptospiral 16S rRNA amplified $265 \mathrm{bp}$ fragment, and was visualised by gel electrophoresis (Fig. 1). Blast sequence analysis of the sequences recorded showed 100\% resemblance with Leptospira interrogans serovar Icterohaemorrhagiae, and serovar Australis, revealing the circulation of pathogenic Leptospira in Pakistan. Phylogenetic tree (Fig. 2) revealed that $L$. interrogans detected in our study was closely related to sequences reported from India, a country neighbouring to Pakistan. The dendrogram shows that the samples studied in this experiment and already reported sequences of intermediate species lie in different clades, providing no evidence of circulation of intermediate Leptospira species in Pakistan.

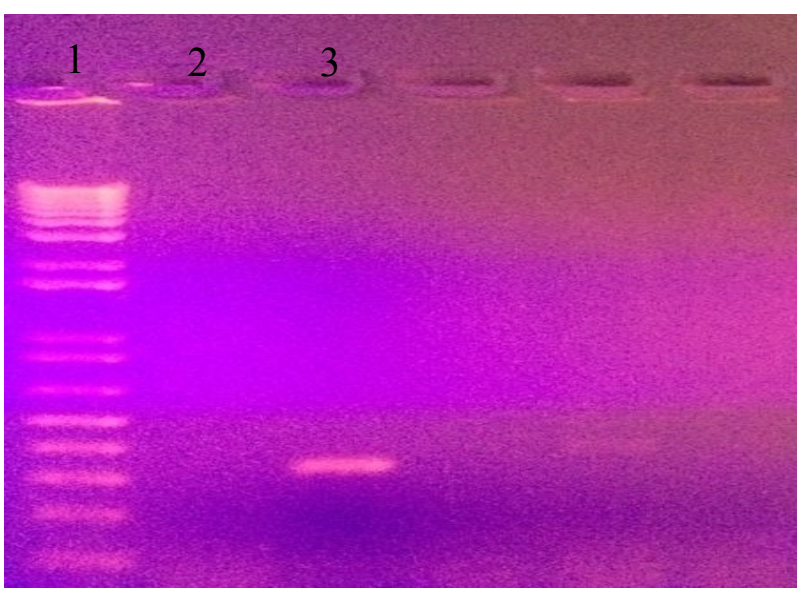

Fig 1. Agarose gel electrophoresis of PCR analysis products for $16 \mathrm{~S}$ rRNA (pathogenic Leptospira, $306 \mathrm{bp})$. Line 1: DNA ladder (1Kb), Line 2: negative sample, Line 3: Positive sample (306 bp)

\section{Discussion}

Leptospirosis is the most widespread zoonosis in the world with higher prevalence in tropical countries. Its clinical signs are nonspecific, making its diagnosis a challenge (29). Despite high rates of seropositivity among animals in Pakistan $(2,20)$, to date, there have been no data on the molecular studies of leptospirosis in horses or in any mammalian host. This investigation reports the first molecular evidence of equine leptospirosis in Pakistan, where previous serological reports have revealed a prevalence of over $40 \%$ (21). 


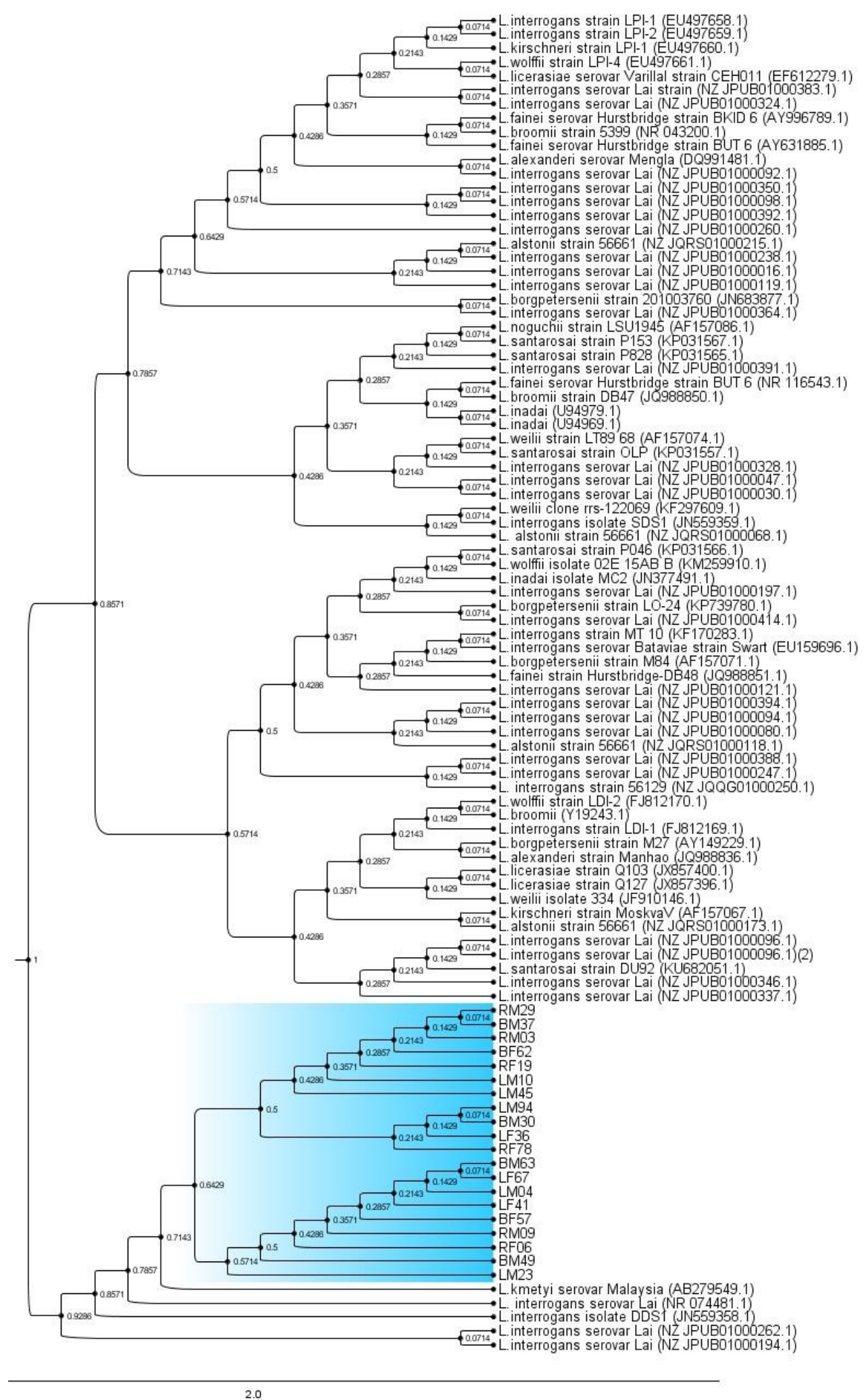

Fig. 2. Maximum likelihood phylogenetic tree based on the nucleotide sequence of Leptospira spp. 16S rRNA in horses. Dendrogram was constructed with 1000 replication using MEGA 6.0 software. The sequences determined in this study are highlighted. GenBank accession numbers of already reported strains are shown in parenthesis

The aim of this investigation was to detect the presence of leptospiral DNA in horse blood samples and to analyse genetic diversity of the Leptospira in the country. Conventional diagnostic tests, such as MAT, are mostly serological and, as such, confirm the disease best at a late acute phase, when antibiotic treatment is already less effective (28). Diagnosis of leptospirosis through culture is not practical in diagnostic laboratories because of the complexity of reagents, several weeks of growth time, and contamination 
problems in culture media (8). All these challenges drew our attention towards the use of molecular techniques for the diagnosis of equine leptospirosis.

The purification, sequencing and phylogenetic analysis of the ribosomal $16 \mathrm{~S}$ gene amplicons revealed that the species involved in infection was Leptospira interrogans (Fig. 2). This confirms that the primer set for standard PCR was specific to pathogenic Leptospira spp. The 16S rRNA is the most common molecular marker for the detection of Leptospira (23), because of its low variability and highly conserved region (15). PCR with the use of these primers is a simple and specific tool for identification of the organisms from different species. Several researchers used 16S rRNA for identification of pathogenic leptospirae (18).

Molecular detection rates were lower in this study as compared to previously reported serological studies in animals $(2,20)$ because the present study explored blood samples for the extraction of leptospiral DNA, where bacteria are found only after 3-10 d exposure. This septicaemic phase is followed by an immune phase (4-30 d) which is characterised by an increased titre of antibodies correlated with the elimination of bacteria from blood $(5,17,18)$.

Prevalence of leptospiral serovars varies depending on particular geographical regions (4). Titres of various serovars have been reported in horses, but Icterohaemorrhagiae, Bratislava, Copenhageni, and Pomona are among the most prevalent serovars (27). Pakistan is a tropical country (22) and in tropical regions serogroup Icterohaemorrhagiae has been reported to be the most frequent infection (3). Blast sequence analysis performed in this study showed $100 \%$ resemblance with $L$. Icterohaemorrhagiae, and $L$. Australis, revealing the circulation of pathogenic Leptospira in Pakistan. Pakistan is bordered by India, Iran, and China and sero-epidemiological studies in these countries used L. interrogans as an antigen for the detection of MAT antibodies, confirming the prevalence of this species in these regions (14). Moreover, $L$. interrogans has been reported to be prevalent in $60 \%$ of leptospirosis cases in china (31). The mapping results of phylogenetic analysis revealed that $L$. interrogans detected in our study is closely related to $L$. interrogans serovar Icterohaemorrhagiae, Automunalis, and Copenhangeni which have also been detected in Iran (6). The phylogenetic tree (Fig. 2) reveals that $L$. interrogans from our study was closely related to the sequences reported from India. Moreover, it reveals that no intermediate species of Leptospira were found in this study. This analysis demonstrates that $L$. interrogans is the prevalent pathogenic species in this region and the use of $16 \mathrm{~S}$ rRNA sequences can be the basis for leptospiral detection in Pakistan.

Being the first ground-breaking study on molecular detection of equine leptospirosis in Pakistan, our research has opened a gateway to future investigation in this area. A further study involving human and environmental samples to explore molecular epidemiology will help to design effective strategies for leptospirosis prevention.

Conflict of Interests Statement: The authors declare that there is no conflict of interests regarding the publication of this article.

Financial Disclosure Statement: This work was financially supported by the Higher Education Commission (HEC), Islamabad, Pakistan.

Animal Rights Statement: Formal consent from the owners of the horses was obtained prior to the collection of blood samples. The study design was submitted to and approved by the Ethical Review Committee for the Use of Animals, UVAS, Lahore, Pakistan.

\section{References}

1. Alvarado-Esquivel C., Hernandez-Tinoco J., Sanchez-Anguiano L.F., Ramos-Nevarez A., Cerrillo S.S.M.L., Saenz-Soto L.: High seroprevalence of Leptospira exposure in meat workers in Northern Mexico: a case-control study. J Clin Med Res 2016, 8, 231-236.

2. Anwar K., Khan N., Mujtaba M.: Seroprevalence of leptospirosis in aborted dairy cattle in Peshawar district Suburb, Khyber Pakhtunkhwa Pakistan. Int J Curr Microbiol App Sci 2013, 2, 73-78.

3. Arent Z., Gilmore C., Brem S., Ellis W.A.: Molecular studies on European equine isolates of Leptospira interrogans serovars Bratislava and Muenchen. Infect Genet Evol 2015, 34, 26-31.

4. Balamurugan V., Gangadhar N.L., Mohandoss N., Assadi S.R., Dhar T.M., Shome R., Krishnamoorthy P., Prabhudas K., Rahman H.: Characterization of leptospira isolates from animals and humans: phylogenetic analysis identifies the prevalence of intermediate species in India. Springerplus 2013. 2, 362-370.

5. Bourhy P., Bremont S., Zinini F., Giry C., Picardeau M.: Comparison of real-time PCR assays for detection of pathogenic Leptospira spp. in blood and identification of variations in target sequences. Clin Microbiol 2011, 49, 2154-2160.

6. Dehkordi J.A., Shahbazkia H.R., Ronagh N.: Evaluation of pathogenic serovars of Leptospira interrogans in dairy cattle herds of Shahrekord by PCR. Iran J Microbiol 2011, 3, 135-139.

7. Doosti A., Ahmadi R., Arshi A.: PCR detection of leptospirosis in iranian camels, Bulg J Vet Med 2012, 15, 178-183.

8. Erol E., Jackson C.B., Steinman M., Meares K., Donahoe J., Kelly N., Locke S., Smith J.L., Carter C.N.: A Diagnostic evaluation of real-time PCR, fluorescent antibody, and microscopic agglutination tests in cases of equine leptospiral abortion. Equine Vet J 2015, 47, 171-174.

9. Filho R.B.O., Malta K.C., Oliveira M.B.J., Santana V.L.A., Harrop M.H.V., Stipp D.T., Pinheiro J.W.J.: Epidemiological analysis of leptospira spp. Infection in equids from the Brejo Paraibano microregion of Brazil. J Equine Vet Sci 2013, 34, 407-411.

10. Galloway R.L., Hoffmaster A.R.: Optimization of lip132 PCR assay for increased sensitivity in diagnosing leptospirosis. Diagn Microbiol Infect Dis 2015, 82, 199-200.

11. Haggag Y.N., Samaha H.A., Nossair M.A., El-Shafii S.S.A., Abdalla S.T.A.: Seroprevalence of Leptospira hardjo in equine and human in Behera Province, Egypt. Alexandria J Vet Sci 2015, 47, 113-118.

12. Hamond C., Martins G., Loureiro A.P., Pestana C., Ferreira R.L., Medeiros M.A., Lilenbaum W.: Urinary PCR as an increasingly 
useful tool for an accurate diagnosis of leptospirosis in livestock. Vet Res Commun 2014, 38, 81-85.

13. Hamond C., Pestana C.P., Medeiros M.A., Lilenbaum W.: Genotyping of Leptospira directly in urine samples of cattle demonstrates a diversity of species and strains in Brazil. Epidemiol Infect 2016, 144, 72-75.

14. Mitra J., Chowdhury S., Pattanayak S.: Sero-prevalence of bovine leptospirosis in south anandaman islands, India. Explor Anim 2015, 5, 96-101.

15. Musso D., Scola B.L.: Laboratory diagnosis of leptospirosis: a challenge. J Microbiol Immunol Infect 2013, 46, 245-252.

16. OIE. Manual of Diagnostic Tests and Vaccines for Terrestrial Animals, World Organisation for Animal Health, Paris, 2016.

17. Picardeau M.: Diagnosis and epidemiology of leptospirosis. Med Mal Infect 2013, 43, 1-9.

18. Prameela D.R., Sreenivasulu D., Seenivasan N.N., Vijayachari P., Lakshmi S.V.: 16S rRNA PCR for characterization of leptospira from andhra pradesh. Int J Sci Environ Tech 2016, 5, 84-94.

19. Rojas P., Monahan A.M., Schuller S., Miller I.S., Markey B.K., Nally J.E.: Detection and quantification of leptospires in urine of dogs: A maintenance host for the zoonotic disease leptospirosis. Eur J Clin Microbiol Infect Dis 2010, 29, 1305-1309.

20. Saleem M.H., Khan M.S., Khan M.A., Khan M.A., Ijaz M., Hassan A., Mehmood K.: Serosurveillance of canine leptospirosis under different climatic conditions in and around Lahore, Pakistan. Pak Vet J 2013, 33, 241-243.

21. Sohail M.L., Khan M.S., Avais M., Zahoor M.Y., Ijaz M., Ullah A., Fatima Z., Naseer O., Khattak I., Ali S.: Seroprevalence of Leptospira spp. in horses of distinct climatic regions of Punjab, Pakistan. J Equine Vet Sci 2016, 44, 82-89.

22. Soomro F.R., Pathan G.M., Gurbakhshani A.L., Kakar J.K.: Prevalence of malarial parasites in Larkano district, Sindh, Pakistan. GJMS 2010, 8, 146-148.
23. Sumanta H., Wibawa T., Hadisusanto S., Nuryati A., Kusnanto H.: Genetic variation of Leptospira isolated from rats catched in Yogyakarta Indonesia. Asian Pac J Trop Med 2015, 8, 10-13.

24. Tadich T.A., Tapia C., González D.: Seroprevalence of Leptospira spp. in working horses located in the Central Region of Chile. J Equine Vet Sci 2016, 38, 14-18.

25. Tilahun Z., Reta D., Simenew K.: Global epidemiological overview of leptospirosis. Int J Microbiol Res 2013, 4, 09-15.

26. Toyokawa T., Ohnishi M., Koizumi N.: Diagnosis of acute leptospirosis. Expert Rev Anti Infect Ther 2011, 9, 111-121.

27. Tsegay K., Potts, A.D., Aklilu N., Lddototter C., Gummow B.: Circulating serovars of Leptospira in cart horses of central and southern Ethiopia and associated risk factors. Prev Vet Med 2016, http://dx.doi.org/10.1016/j.prevetmed.2016.01.009.

28. Vanasco N.B., Jacob P., Landolt N., Chiani Y., Schmeling M.F., Cudos C., Tarabla H., Lottersberger J.: Diagnostic accuracy of an igm enzyme-linked immunosorbent assay and comparison with 2 polymerase chain reactions for early diagnosis of human leptospirosis. Diagn Microbiol Infect Dis 2016, 84, 292-297.

29. Ye C., Yan W., McDonough P.L., McDonough S.P., Mohamed H., DiversT.J.: Serodiagnosis of equine leptospirosis by enzymelinked immunosorbent assay using four recombinant protein markers. Clin Vaccine Immunol 2014, 21, 478-483.

30. Zala D.B., Khan V., Das V.K.: A study on few biochemical parameters of clinically suspected and laboratory confirmed leptospirosis cases. J Appl Nat Sci 2014, 6, 12-13.

31. Zhang C., Wang H., Yan J.: Leptospirosis prevalence in Chinese populations in the last two decades. Microb Infect 2012, 14, 317-323. 